\title{
SUR L'UTILISATION DU SULFATE DE CUIVRE EN THERAPEUTIQUE PISCICOLE
}

\author{
établie par M. J.-P. GERARD, Docteur Vétérinaire \\ Laboratoire d'ichtyopathologie de I'INRA \\ 78850 THIVERVAL-GRIGNON
}

\section{Description du produit :}

Le sulfate de cuivre se trouve couramment dans le commerce où il est appelé a vitriol bleu». C'est une forme hydratée du produit pur.

II se présente sous l'aspect d'une poudre, ou de cristaux bleus, très solubles dans l'eau.

Sa solubilité dépend de la température de l'eau; elle croit avec la température; elle est de 300 grammes par litre d'eau à $0 \circ$ et de $2000 \mathrm{~g} / \mathrm{litre}$ d'eau à $100^{\circ}$.

\section{Indications :}

Couramment utilisé dans la lutte contre les maladies cryptogamiques en agriculture et viticulture, le sulfate de cuivre est actif contre les myxobactéries, les algues, les champignons et d'une manière générale contre les microparasites externes. Son emploi préventif évite le développement de ces microorganismes. Curativement, il permet de détruire tous ces * parasites * mais les résultats obtenus sont moins spectaculaires, car il ne peut dès son emploi réparer les troubles physiologiques déjà occasionnés par la maladie.

\section{Toxicité :}

Le sulfate de cuivre en bain permanent à $+15^{\circ} \mathrm{C}$ est toxique pour la truite à la dose de $0,14 \mathrm{ppm}$ (1) soit 0,14 gramme par mètre cube.

Les doses thérapeutiques employées sont plus élevées, mais la durée des bains est alors beaucoup plus courte. On peut, dans des conditions précises, employer sans danger ce produit pour traiter les poissons.

La toxicité dépend de la dureté de l'eau. Elle est plus élevée dans les eaux acides et douces que dans les eaux alcalines et dures.

\section{Posologie :}

La toxicité de ce médicament étant liée aux qualités de l'eau, il est difficile de donner une posologie polyvalente.

Avant l'emploi, le pisciculteur devra faire un essai préalable sur quelques poissons, pour juger de leurs réactions. La posologie est lièe à la température de l'eau, et à la durée du traitement. A $140 \mathrm{C}$, la posologie pour une eau contenant 20 à $50 \mathrm{mg} /$ litre de carbonate de calcium sera la suivante :

(1) ppm : partie par million, soit un gramme par mètre cube ou un milligramme par litre. 
- 1 g pour 2 litres d'eau $(1 / 2000)$ - Bain éclair (moins d'une minute).

- $1 \mathrm{~g}$ pour 10 litres d'eau $(1 / 10000)$ - Bain de 10 minutes environ.

- ou dose arbitraire utilisée dans le courant d'eau suivant la technique décrite dans le mode d'emploi ci-après (B. 2).

\section{Mode d'emploi :}

\section{A) Bain concentré :}

Il s'effectuera de préférence à l'occasion d'un tri des animaux, car ce mode de traitement $\in$ n lui-même est fastidieux pour l'opérateur et peut être dangereux pour des poissons déjà malades.

Les sujets, placés dans une épuisette, sont immergés dans la solution de sulfate de cuivre à 1 pour 2000 pendant un temps inférieur à 30 secondes.

\section{B) Bain dilué :}

\section{1 - Traitement dans les auges d'alevinage.}

Le volume d'eau dans l'auge est d'abord déterminé. Une solution concentrée qui permettra d'obtenir dans l'auge la concentration finale de 1/10 000 est préparée dans un arrosoir. La solution concentrée contiendra autant de grammes de sulfate de cuivre qu'il y a de fois 10 litres d'eau dans l'auge d'alevinage. L'arrivée d'eau étant arrêtée, la solution concentrée est répartie dans l'auge puis brassée à l'aide d'un balai pour obtenir une solution bien homogène. Après 10 minutes les $2 / 3$ de l'eau sont vidangés et l'arrivée de l'eau rétablie simultanément.

\section{2 - Traitement dans les bassins.}

Une quantité de sulfate de cuivre ( 300 à $500 \mathrm{~g}$ ) sous la forme de cristaux, car les cristaux se dissolvent moins rapidement dans l'eau que la poudre, est placée dans un linge épais et ligaturé en forme de sac, avec une ficelle. $\mathrm{Ce}$ sac est plongé dans l'eau à l'entrée du bassin et solidement attaché par la ficelle. Le sulfate de cuivre se dissout dans l'eau. Les poissons fuient la vague médicamenteuse qui progresse vers l'aval. Lorsque tous les poissons sont réunis dans le quart aval du bassin, le sac est retiré de l'eau. Cette technique est la plus intéressante à employer, car elle évite toute manipulation de poisson.

Lorsque les bassins sont en série, on peut traiter les poissons en utilisant cette technique à condition de commencer le traitement par le bassin situé en amont.

Dans le premier bassin amont le sac contenant le sulfate de cuivre est immergé dans l'arrivée d'eau. Les poissons fuient la vague médicamenteuse et le traitement sera arrêté dès qu'ils auront atteint le quart aval du bassin. L'eau chargée en sulfate de cuivre arrivera dans le bassin suivant, elle déclenche la fuite des poissons. Si la dose est insuffisante, l'opérateur peut placer à l'entrée de ce bassin le sac contenant le sulfate de cuivre, il le retirera lorsque les poissons auront fui et atteint le quart aval, il procèdera ainsi de bassin en bassin. II est possible qu'en troisième ou en quatrième eau, il ne soit plus utile d'ajouter du sulfate de cuivre. L'intérêt de cette technique repose sur le fait que de l'eau non chargée en sulfate de cuivre arrive immédiatement après avoir retiré le sac contenant ce produit.

\section{Périodicité :}

Les bains de sulfate de cuivre peuvent être effectués :

- préventivement tous les 10 à 15 jours.

- curativement deux bains à 24 heures d'intervalle, un troisième 48 heures après, puis un tous les 10 à 15 jours. 